\title{
Interactive comment on "Comparing the impact of environmental conditions and microphysics on the forecast uncertainty of deep convective clouds and hail" by Constanze Wellmann et al.
}

\section{Anonymous Referee \#1}

Received and published: 12 August 2019

Review of "Comparing the impact of environmental conditions and microphysics on the forecast uncertainty of deep convective clouds and hail" by Wellmann et al.

This study examines the relative influence of environmental conditions and microphysical parameters on vertically integrated hydrometeor contents and precipitation values (with an emphasis on hail), in addition to their influence on heating rates. The authors use an emulator technique to reduce the number of simulations that would otherwise be required to produce the presented results. In general, the study finds that environmental conditions and microphysical properties contribute to forecast uncertainty; however, when the environment and microphysics interact with each other, the latter

Printer-friendly version

Discussion paper 
tends to dominate forecast uncertainty in hydrometeor contents and precipitation.

Overall, I have a long list of comments; most importantly, I think that the authors should provide more analysis and discussion in several of the sections (please see general and specific comments). The paper is well written, but readability could be improved by a more liberal use of commas in addition to the rewording of several sentences (please see specific comments). In addition, the authors should try to be consistent about their use of active versus passive voice. With all of this said, I think that the results are interesting and worthy of publication, and at this stage I suggest acceptance subject to major revision.

Major/general comments:

1. Model Setup (Section 2) and Methods (Section 3): For clarity, and especially for those readers who are not familiar with the emulator technique, there should probably be more information included about the modeling approach. For example, what is the total number of simulations conducted? Which "input combinations" are simulated? How does your choice for the mean function and correlation structure influence the results? How do you validate the emulator? Are the emulator results sensitive to the chosen minimum and maximum parameter values? What is the argument for including CCN and INP in the environmental conditions setup? Do the results change notably if these two microphysical properties are included only in the microphysical conditions setup?

2. Sensitivity Analysis for Variations of the Microphysics (S2) (Section 4): This section, which represents the bulk of the paper, generally lacks depth and therefore should contain additional insight and discussion. For instance: [P9, L14-20] Why look at the mean for hydrometeor content, max for precipitation, and both for amount of hail? [P9, L31-32] What about snow and hail as contributors to the output uncertainties? Maybe this should say one of the largest contributors. [P9, L39-40] Total precipitation, which is a very important quantity, seems to be affected more notably by the fall speed of

Printer-friendly version

Discussion paper
Interactive

comment 
graupel scaling factor than by CCN. Please comment on this.

3. Heating rates (Section 4.2): The results from this section are quite interesting; however, I feel as though it is lacking a bit in terms of analysis and discussion. It would be nice if the authors took some time to dive a little deeper. For example: [P11, L17-18] Why is the fall speed of graupel the most important at low altitudes $(<2 \mathrm{~km})$ and high altitudes $(>10 \mathrm{~km})$, which is where the graupel heating rates are very small if not zero? Is graupel present in these regions? Perhaps a figure showing vertical profiles of hydrometeor contents may help. [P11, L18-19] Where the fall speed of rain plays a notable role in the main effect (between 3 and $4 \mathrm{~km}$ ), the magnitude of the rain heating rate is only a small fraction of the magnitude of the total heating rate. Does this suggest that, in general, the model physics is more uncertain about rain evaporation processes than cloud condensation processes? [P12, L2-3] Can you speculate as to why the CCN concentration and fall speed of graupel dominate the total heating rate output uncertainty at high altitudes?

4. Hydrometeor masses and precipitation (Section 5.1): In general, this section would benefit from a deeper (and more quantitative) analysis. Figure 4 is really nice for visual comparison; however, can the numerical values be put into a table (perhaps in an appendix or a supplement) for a more quantitative comparison? Also, to minimize reader confusion, I recommend not putting a circle in areas where the input parameter was not part of the emulator simulation. For instance, under S2 for shear, under S1 for graupel fall speed, etc. Some comments and questions about the section text: [P14, L4-6] When referring to the trend for precipitation output, what about for total precipitation rate, which appears to be different? Is this important? Again, a table would help clarify these comparisons. [P15, L3] When referring to the main effect of the INP concentration, what is the physical interpretation? The influence of uncertainty in the INP concentration is muddled when the uncertainty in the other (individual? some? all?) microphysical parameters are introduced? Please elaborate. 
1. P1, L15-17: Maybe reword to make more clear that you are emphasizing environmental parameters and microphysical parameters. Also, please separate the citations to better associate with these two different aspects of forecasting convective clouds.

2. P2, L3: What are the different choices of the trigger? 3. P2, L5-6: Please add references for the Morrison and Thompson schemes.

4. P2, L6-7: Which aspects of the parameterizations are most influential?

5. P2, L8: Individual parameters such as?

6. P4, L7: Horizontal resolution or grid spacing?

7. P4, L9: Can you provide approximate vertical grid spacings in the layer(s) of interest?

8. P4, L9: Do the open boundary conditions cause any mass conservation issues?

9. P4, L20: How are cloudy grid boxes defined?

10. P4, L26: Which "two former studies"?

11. P5, Table 1: The fourth input says "potential temperature at the ground", but the text says "vertical temperature profile". Please clarify.

12. P5, L6: Perhaps "maritime" should be changed to "clean" because marine cloud can be polluted.

13. P5, L17-18: This sentence is confusing...does it turn toward the west until a straight easterly flow is reached or does it turn toward the east until a straight westerly flow is reached?

14. P5, L20: Please state explicitly the wind direction bounds at the surface.

15. P5, L20: Please add a citation for this statement.

16. P5, L23: Please add a citation for this statement. 
17. Table 2: Please add the symbol/abbreviation for the various parameter inputs (where necessary).

18. P7, L11: Why not also vary $\mu$ ?

19. P7, L32: Please change "data" to "output".

20. P8, L20-21: Can you comment on the errors that are associated with this prediction?

21. P9, L16: Contents or vertically integrated contents?

22. Figure 2: Is it possible to coordinate the $y$-axes of the two panels so that a direct comparison in the vertical is easier?

23. P11, L13-15: While this is true, perhaps note that the total heating rate does not decrease as rapidly as the rate due to cloud water because graupel and ice heating rates are at a maximum between about 8 and $10 \mathrm{~km}$.

24. P12, L31-33: The impact of the fall speed of hail and the strength of ice multiplication is mentioned as being important, but what about the role of the fall speed of graupel? This seems quite important for small hail. Moreover, can you speculate as to why CCN concentration becomes more important at larger hail diameters?

25. Table 3: What is the difference between assigning the input parameters "lower" and "higher" values as opposed to "min" and "max" values (as in Tables 1 and 2)? Also, why are the values used in this experiment different from those used for S1 and S2? Is the input listed as "potential temperature" at the surface?

26. P14, L1 (below Fig. 4): Can you parenthetically reference the CCN and INP concentrations here to help the reader? 27. P15, L4-7: Maybe note that, in general, uncertainties in wind shear (which is likely not uncommon in numerical weather prediction) do not have a notable impact on the output variables examined here with the exception of integrated rain water content (rain water path). 
28. P15, L6: Perhaps note that the impact of theta is already relatively low.

29. P15, L31-32 ("The remaining input parameters [...]"): I am not sure that I underACPD stand this sentence. Please clarify.

30. P15, L34-35 ("in particular from the fall velocity of graupel for the hydrometeor masses and from the fall velocity of hail for precipitation"): This seems like an important finding; please italicize for emphasis.

31. P15, L35-36: So, are you able to say that uncertainties in the selected environmental conditions are muddled by uncertainties in the selected microphysical parameters?

32. P15, L50-51 ("In their study [...]"): This sentence is a bit confusing; please reword and/or flesh out.

33. P16, L7: To which aerosol effect(s) are you referring?

34. P16, L8-11: Can you be more specific about the Fan et al. (2013) results? Changes up to $25 \%$ ? $25 \%$ on average? Is their range of CCN concentration similar to the one used here? Also, for the Yang et al. (2017) paper, what range of CCN concentration was tested? This is important when comparing previous results to results from the current study.

35. Figure 5: For the left panel, please mark the $0 \mathrm{~K} / \mathrm{h}$ value to delineate between negative and positive values. Also, for both panels, is it possible to make the lines thicker in this figure (as in Fig. 2)?

36. P17, L17-18: When talking about the saturation adjustment in the microphysics scheme, how are you able to make this conclusion? Please elaborate.

37. P17, L33-34: When talking about the emulator-predicted size distributions, are you able to comment on the expected uncertainty in your results?

38. Figure 6: As in Fig. 5, are you able to make the lines thicker? 
39. Size distribution of surface hail (Section 5.3): For the analysis in this section, please use line color and style to help clarify to which distribution you are referring.

40. P18, L5: When referring to the value of $0.4 \mathrm{~mm}-1 \mathrm{~m}-3$, this number does not seem to correspond with the y-axis. Am I missing something here?

41. P18, L7: Similar to comment \#40, when referring to the value of $3.4 \mathrm{~mm}-1 \mathrm{~m}-3$, this number does not seem to correspond with the $y$-axis.

42. P18, L7-9: This sentence is confusing. Perhaps say something like: "when relatively high (low) values of theta and high (low) concentrations of CCN and INP are present, low (high) hail number concentrations result".

43. P18, L10-12: This could probably be stated more simply in one sentence. For example, something like: "For S2, the maximum and minimum of the hail size distribution is larger and smaller than that for $\mathrm{S} 1$, leading to a larger spread in the distributions."

44. P19, L3-10: What about for larger diameters? It is especially interesting that the number concentrations for the minimum size distributions are largest for S3. This suggests that, individually and for the lower bound, S1 and S2 do not produce large number concentrations, but if the environmental and microphysical conditions are combined (S3), then there is an enhancement. Please comment on this notable difference.

45. P19, L8-10: So, can you speculate as to what this means physically?

46. P19, L12-13 ("While the microphysical input parameters mainly determine the maximum number concentration, the environmental conditions substantially influence the minimum number concentration"): This seems like an important finding; please italicize for emphasis. 47. P20, L17-27: There is a large body of literature, some of which is referenced in the introduction, that focuses on the so-called "aerosol invigoration" hypothesis. Can you relate the work presented here to previous work?

48. P21, L19-20: What about also a revised parameterization of the fall speed of graupel? 
Grammatical/wording recommendations:

1. The authors interchange hydrometeor "content" and "mass" throughout the text. Which parameter is actually shown? Please be consistent.

2. P2, L4-5: Awkward sentence; please reword.

3. P2, L5: Change "three cloud types for" to "three cloud types using".

Interactive

comment

4. P2, L19: Maybe "Additional relevant" instead of "Further relevant"?

5. P2, L28: Change "Moreover, field studies indicate that fall speeds of hydrometeors are observed in a broad range of velocities." to "Moreover, field study observations indicate that hydrometeors may have a broad range of fall velocities."

6. P2, L30-32: Awkward sentence; please reword.

7. P3, L10-12: Awkward sentence; please reword.

8. P3, L12: No need for "different".

9. P3, L13: Change "sometimes thermodynamic conditions are the main drivers, sometimes dynamic conditions" to "either thermodynamic conditions or dynamic conditions may be the main driver".

10. P8, L31: "in the Fourier space along all parameters change simultaneously" is awkward; please reword.

Interactive comment on Atmos. Chem. Phys. Discuss., https://doi.org/10.5194/acp-2019-558, 2019. 